\title{
Well-being of Academy Fellows and specialty trainees: what is the problem?
}

This article was published on 5 Oct 2021 at www.hkmj.org.

WC Leung ${ }^{1 *}$, MD, FHKAM (Obstetrics and Gynaecology), Rosalie Lo², PsyD, FHKPS, Jeremy YC Teoh ${ }^{3}$, FRCSEd (Urol), FHKAM (Surgery), Aaron Cheng ${ }^{4}$, MSc, FRSPH, Martin CS Wong ${ }^{5}$, MD, MPH, Gilberto KK Leung ${ }^{6}$, MB, BS (Lon), FHKAM (Surgery); for the Task Force on Well-being of the

Hong Kong Academy of Medicine

${ }^{1}$ Honorary Secretary, Hong Kong Academy of Medicine

${ }^{2}$ Honorary Advisor, Task Force on Well-being, Hong Kong Academy of Medicine

${ }^{3}$ Chair, Young Fellows Chapter, Hong Kong Academy of Medicine

${ }^{4}$ Chief Executive Officer, Hong Kong Academy of Medicine

${ }^{5}$ Editor, Hong Kong Academy of Medicine

${ }^{6}$ President, Hong Kong Academy of Medicine

*Corresponding author: leungwc@ha.org.hk

Hong Kong Med J 2021;27:324-5

https://doi.org/10.12809/hkmj215123

In this issue of Hong Kong Medical Journal, Kwan et $\mathrm{al}^{1}$ report the results of a survey of young doctors in Hong Kong (residents in training and specialists within 10 years of registration), showing high rates of burnout using the Copenhagen Burnout Inventory (73\% reported personal burnout; $71 \%$ reported work-related burnout; and 55\% reported client-related burnout) and depression using the Patient Health Questionnaire-9 (21\%). Although the worldwide prevalence of burnout among medical professionals is increasing, ${ }^{2}$ the local situation in Hong Kong ${ }^{1,3,4}$ is alarming and the Hong Kong Academy of Medicine is committed to tackling this problem. A Well-being Charter has been promulgated in confirmation of the commitment of the Academy and its 15 constituent Colleges.

\section{Hong Kong Academy of Medicine Well-being Charter}

The Academy is mandated and empowered to promote and advance healthcare for Hong Kong citizens and foster a spirit of cooperation among medical and dental practitioners.

Quality patient care and doctors' well-being go hand in hand, and the Academy is committed to assuming a leading role in cultivating and promoting the well-being of Fellows and trainees of its constituent Colleges as a shared responsibility among individual doctors, respective employing institutions, and policy makers.

The Academy shall:

- acknowledge the importance of well-being of medical and dental practitioners in contributing towards high quality and effective patient care;

- prioritise Fellows' and trainees' well-being as a prerequisite for fulfilling their professional duties towards patients and the community; promote the well-being of its Fellows and trainees as a necessary condition for their flourishing and whole-person development;

- foster a caring and supportive culture within the healthcare professions;

- strengthen professionalism across disciplines and foster interprofessional collaboration from the institutional perspective;

- encourage and facilitate healthcare practitioners to attend to their physical, mental, and social health and to respond to burnout, stress, or emotional challenges promptly and proactively;

engage a network and establish mechanisms to provide up-to-date knowledge, self-care tools, peer support and professional assistance for medical and dental practitioners;

- organise mental health training and embed awareness of well-being in training and continuous professional development for medical and dental practitioners;

promulgate good practices among institutions to build supportive systems at the organisational level through the establishment of effective communication channels, deployment of appropriate resources, and quality improvement strategies; and

advocate policy changes and propose initiatives to the Government and other policy makers on professional well-being issues and the alignment of values and practices for the betterment and well-being of the profession.

The Academy has set up a Task Force on Well-being, involving an Honorary Advisor ( $\mathrm{R}$ Lo), the Academy Officers and secretariat staff, as well as representatives from the Social Subcommittee, the Young Fellows Chapter and the 15 Colleges. In line with the Charter, the Task Force on Well-being is following an incremental ASAP (Awareness; 
Self-care; Ask for help; Promotion of well-being) approach to promote well-being and to manage stress and burnout.

\section{Awareness}

- Helping you understand and recognise the causes, signs, and symptoms of burnout, stress, and other mental well-being issues

\section{Self-care}

- Providing practical advice for enhancing wellbeing and managing stress

\section{Ask for help}

- Facilitating a peer support network for you

- Providing information on getting professional help

\section{Promotion of well-being}

- Cultivating a culture of care and support among Fellows

- Making recommendations for well-being improvement at the organisational level

- Organising or promoting well-being programmes and activities

Further details will be available on a dedicated page on the Academy website (https://well-being. hkam.org.hk/) to facilitate this ASAP approach.

\section{Author contributions}

All authors contributed to the concept or design; acquisition of data; analysis or interpretation of data; drafting of the article; and critical revision for important intellectual content. All authors had full access to the data, contributed to the study, approved the final version for publication, and take responsibility for its accuracy and integrity.

\section{Conflicts of interest}

All authors have disclosed no conflicts of interest.

\section{References}

1. Kwan KY, Chan LW, Cheng PW, Leung GK, Lau CS; for the Young Fellows Chapter of the Hong Kong Academy of Medicine. Burnout and well-being in young doctors in Hong Kong: a territory-wide cross-sectional survey. Hong Kong Med J 2021;27:Epub 5 Oct 2021.

2. The Lancet. Physician burnout: a global crisis. Lancet 2019;394:93

3. Siu CF, Yuen SK, Cheung A. Burnout among public doctors in Hong Kong: cross-sectional survey. Hong Kong Med J 2012;18:186-92.

4. Ng AP, Chin WY, Wan EY, Chen J, Lau CS. Prevalence and severity of burnout in Hong Kong doctors up to 20 years post-graduation: a cross-sectional study. BMJ Open 2020;10:e040178 